\title{
El Tucídides de Heredia en el contexto de las antologías medievales de discursos historiográficos
}

Juan Carlos Iglesias-Zoido

Universidad de Extremadura

El objetivo de nuestro artículo es analizar el contexto retórico de las antologías de discursos historiográficos que circularon en la baja Edad Media para comprender la auténtica naturaleza y finalidad del Tucídides de Heredia. ${ }^{1}$ De este modo, buscamos explicar los motivos últimos que llevaron a la elaboración de este tipo de antologías, el modo en que se utilizaron y las diferentes aplicaciones prácticas que recibieron a finales del siglo XIV.

\section{EI Tucídides de Heredia: una antología de discursos historiográficos}

Entre las obras redactadas en el scriptorium dirigido por Juan Fernández de Heredia en el Avignon de la segunda mitad del siglo XIV llama poderosamente la atención aquella que suele denominarse como el Tucídides de Heredia. ${ }^{2}$ A pesar del título por el que tradicionalmente es conocida, no se trata de una copia completa de la Historia de la Guerra del Peloponeso, la gran obra escrita por el historiador griego Tucídides a finales del siglo $\mathrm{V}$ a.C., sino que el lector que toma

\footnotetext{
${ }^{1}$ Este estudio se enmarca en el proyecto de investigación BFF2012-31813: "Las selecciones de discursos de origen historiográfico II”. Una primera aproximación a este tema se presentó en el Congreso sobre Humanismo y Tradición Clásica celebrado en Alcañiz en el año 2005 y está publicada en Iglesias-Zoido (2008). El presente artículo ofrece una actualización en la que hemos centrado la atención en el contexto de las antologías de discursos de origen historiográfico.

${ }^{2}$ La edición del texto puede consultarse en Álvarez Rodríguez (2007). Con respecto a esta obra de Heredia, cf. nuestros estudios previos: Iglesias-Zoido (2005), (2008), (2011: 135-154) y (2015).
} 
en sus manos el manuscrito 10.801 de la Biblioteca Nacional se encuentra ante una antología que recoge la mayor parte de sus discursos. Sólo este hecho, el que se trate de una antología de discursos historiográficos, ya conferiría un valor especial a esta obrita, al ofrecernos el resultado de una labor selectiva realizada en el marco del scriptorium del Gran Maestre en Avignon. Sin embargo, su importancia se acrecienta aún más si tenemos en cuenta que no se trata de una selección en lengua original que nos permita atestiguar la influencia del historiador ateniense en la Europa de este momento, sino que estamos ante una traducción al aragonés castellanizado que se empleaba en el estudio del Gran Maestre. Una característica que le confiere al Tucídides el honor de ser la primera traducción a una lengua vernácula de una parte esencial de esta historia decisiva para la cultura de Occidente. Esto significa que, antes de la primera y enormemente influyente versión al latín realizada por Lorenzo Valla en 1452, existió una traducción parcial en el ámbito cultural del Aragón de la segunda mitad del siglo XIV. Un dato que apenas había sido señalado hasta hace poco ni en las obras dedicadas a describir la influencia de la tradición clásica en la baja Edad Media ni en aquellas que tenían como objetivo el estudio del legado de Tucídides. ${ }^{3}$ Pero un dato que, en definitiva, merece ser destacado y que otorga al ámbito hispánico un jalón muy importante en la historia de la recepción de los textos clásicos. ${ }^{4}$

El hecho de que se trate de una antología de discursos historiográficos traducida al aragonés plantea cuestiones a las que la crítica ha intentado aportar respuestas. Entre ellas se destacan sobre todo dos: el proceso de elaboración de esta traducción y su finalidad. A la primera cuestión, autores como Lutrell y Álvarez Rodríguez han dedicado páginas fundamentales que han permitido ir trazando las diversas etapas por las que pudo pasar el proceso de traducción. ${ }^{5} \mathrm{Y}$, en nuestro caso, hemos dedicado ya varios trabajos en los que hemos analizado una serie de características codicológicas que, desde nuestro punto de vista, ponen de manifiesto que estamos ante la traducción al aragonés de una antología previa que circularía en Bizancio y que fue tomada como modelo. ${ }^{6}$ En el presente artículo, nuestro interés se centra en la segunda cuestión: la finalidad de esta antología. Descartados otros objetivos de tipo anticuario, ya Cacho Blecua avanzó hace años como elemento clave la finali-

\footnotetext{
${ }^{3}$ Cf. Pade (2003) y (2006).

${ }^{4}$ Cf. Iglesias-Zoido (2011: 135-154) y (2015).

${ }^{5}$ Cf. Lutrell (1960) y Álvarez Rodríguez (2007: XXVIII-XXI).

${ }^{6}$ Cf. Iglesias-Zoido (2005) y (2011: 135-154).
} 
dad retórica. ${ }^{7}$ De hecho, ésta es la única que permite explicar de manera totalmente convincente las causas por las que, a finales del siglo XIV, se planteó la elaboración del manuscrito de lujo en el que se copiaron juntas la traducción al aragonés de los discursos del historiador griego Tucídides y las alocuciones extraídas de la Historia Troyana de Guido delle Colonne. ${ }^{8}$ Pero hablar de "finalidad retórica" no permite comprender en toda su extensión las características y el alcance de este texto sin tener en cuenta el contexto técnico en el que se elaboró: el hecho de que se trate de una antología de discursos historiográficos, el modo en que están dispuestos, a qué necesidades concretas respondía, cómo podían ser empleados en la práctica, etc. Por este motivo, a lo largo de las páginas siguientes, vamos a enmarcar el Tucídides de Heredia en el contexto de las prácticas retóricas que convirtieron a las antologías de discursos de origen historiográfico en un producto cultural de primer orden. Este estudio nos va a obligar a tomar en consideración las diferentes formas en que se manifestó la difusión, uso e influencia de las antologías de discursos en la Europa de la baja Edad Media, para entender en última instancia cuál fue la causa que permite explicar que en el scriptorium de Heredia se llevase a cabo la copia de una obra tan especial como ésta.

\section{El interés por los discursos historiográficos en la Europa bajomedieval}

A lo largo de la baja Edad Media, pero sobre todo en los siglos XIV y XV, se extiende por toda Europa un enorme interés por los discursos de los historiadores de la Antigüedad en los que se transmitía para la posteridad las palabras pronunciadas por grandes generales y estadistas del pasado como Pericles, Alejandro, César o Augusto. El acceso a los discursos de los héroes romanos estaba garantizado por una tradición cultural que había mantenido intacta la admiración por historiadores como Julio César o Salustio, cuyas obras sobre los turbulentos años del final de la República habían gozado de una amplia difusión y no habían dejado de ser copiadas en las bibliotecas de los monasterios de Occidente. ${ }^{9}$ Unas alocuciones en prosa que eran complementadas con la lectura de los discursos que un autor épico como Lucano había insertado en su Farsalia, poema que para

\footnotetext{
${ }^{7}$ Cf. Cacho Blecua (1991) y Gómez Moreno (1996). Con respecto a otras finalidades, cf. López Molina (1960: 45).

${ }^{8}$ Cf. Sanz Julián (2012).

${ }^{9}$ Cf. Osmond y Ullery (2003).
} 
el hombre medieval constituía una fuente de información privilegiada sobre el terrible período de luchas civiles vivido al final del siglo I a.C. No importaba que las palabras de César o de Pompeyo recogidas por Lucano estuvieran en verso si, de este modo, los lectores podían aproximarse a los argumentos que pudieron ser esgrimidos por los protagonistas de un período fundamental de la historia antigua. Algo que, por otra parte, ya ocurría en Roma, donde la obra de Lucano jugó un papel destacado como modelo en las escuelas de retórica desde la época imperial. ${ }^{10}$

Incluso autores casi olvidados de la literatura latina, como Quinto Curcio, volvieron a vivir una auténtica edad de oro en los siglos del final de la Edad Media gracias a los numerosos discursos que incluían. De hecho, aunque la obra de Curcio no dejó de ser leída, se daba la circunstancia de que la historia de Alejandro había sido transmitida sobre todo por otros intermediarios posteriores que añadieron abundantes motivos novelescos al relato histórico. Fue precisamente a partir del XIV cuando la obra de Curcio volvió a ser tenida realmente en cuenta y valorada como más fidedigna que las obras medievales de contenido más novelesco. ${ }^{11}$ Sobre todo, gracias a que en su historia, enormemente influida por la retórica de época imperial romana, se prestaba una especial atención a los discursos que Alejandro habría pronunciado antes de las batallas de Iso y Gaugamela, que convirtieron al rey macedonio en el dueño y señor de Asia y en modelo de líder militar y político. La fascinación por el macedonio, que había generado toda una riquísima tradición medieval de obras dedicadas a glosar su vida y hazañas, se centra ahora en obras históricas de la antigüedad clásica, como la historia de Curcio, que trataban más su figura de manera más realista y que, sobre todo, transmitían sus vigorosos discursos. ${ }^{12}$

\footnotetext{
${ }^{10}$ Cf. Bonner (1966).

${ }^{11}$ Cf. Winterbotton en Reynolds (ed.) (1983: 148-149) y Brunhölzl (1986), sobre los avatares de la transmisión medieval del texto de Curcio. Hay que destacar que la influencia de su obra en la Edad Media apenas ha recibido atención, ya que los estudios no han avanzado demasiado desde la obra clásica de Dosson (1887).

${ }^{12}$ Cf. Dosson (1887), quien ya apuntaba la existencia de más de cien manuscritos medievales (pp. 315-356), la mayor parte de ellos de origen francés, entre los que destaca (pp. $365 \mathrm{ss}$.) el interés por los discursos que se percibe en algunos de ellos. Incluso, hay alocuciones de Curcio en excerpta de discursos historiográficos (Salustio, Livio y Curcio). Sobre los manuscritos de Curcio copiados en los siglos XI y XII, cf. Munk Olsen (1982: 355-362).
} 
Es más, el hombre culto de la baja edad media sentía tal interés por este tipo de discursos de la Antigüedad que no tardaron en promoverse traducciones que permitiesen la lectura y disfrute de los historiadores griegos que hasta entonces sólo eran conocidos de manera indirecta. Así, tras siglos en los que la lengua y cultura helenas ocuparon una posición totalmente marginal dentro de los círculos eruditos del Occidente medieval, ${ }^{13}$ a partir del trescientos se asiste a un renovado interés por el conocimiento, traducción y difusión de autores griegos como Plutarco o Flavio Josefo. ${ }^{14}$ En concreto, los discursos de la historia de Josefo, que era una fuente de información de enorme interés con respecto al mundo judío de los primeros años de nuestra era, adquirieron fama como ejemplos de elocuencia y empezaron a ser más apreciados que los insertos en la historia de Hegesipo que, desde la Alta Edad Media, había transmitido a los lectores latinos la esencia de esta historia escrita en griego en la que se relataba la caída de Jerusalén y en la que se destacaban sus discursos. De hecho, este texto latino compuesto hacia el 375 d.C., transmitido en numerosos manuscritos medievales bajo el título De excidio urbis Hierosolymitanae, llegó a ser considerado como una traducción latina libre del texto griego del Bellum Iudaicum de Flavio Josefo. ${ }^{15}$ Y su obra había sido muy valorada por los historiadores del Renacimiento carolingio como fuente de unos discursos que estaban a la par de los presentes en el texto de Salustio. ${ }^{16}$ Pues bien, el interés de contar con los discursos que originalmente había escrito Flavio Josefo en griego también generó un nuevo interés por su obra y, en lo que a nosotros

\footnotetext{
${ }^{13}$ Cf. Weiss (1977).

${ }^{14}$ Una visión de conjunto sobre la influencia de Plutarco en España desde el siglo XIII la ofrece Bergua Cavero (1995). Sobre Flavio Josefo, cf. Coroleu (2001). Un cuadro general de las traducciones de los historiadores griegos durante esta época en Coroleu (2004).

${ }^{15}$ Cf. Ussani (1932).

${ }^{16}$ Cf. Lake (2013, p. 237): "Hegesippus, like Sallust, was deliberately mined for material that could be used for formal orations and rhetorical set pieces"; p. 240 "he prefers to imitate the speeches of Sallust or Hegesippus". De hecho, parece que no tuvo problemas en poner en boca de Carlos de Lorena, que lucho con Hugo Capeto por el trono de Francia en torno al 987-991, un discurso del Rey Herodes a partir del texto de Hegesipo. O, en otro caso, la adaptación con mínimos cambios del discurso de Adherbal al senado romano: "A similar example of Richer's adaptation of material from a historiographical model occurs in a speech from book 2.52 in wich Louis IV complains to his wife and his loyal followers about his persecution at the hands of Hugh the Great".
} 
nos interesa, la elaboración de antologías de sus discursos que tuvieron una gran circulación durante los siglos XIV y XV. ${ }^{17}$

Las Vidas Paralelas de Plutarco, por su parte, fueron muy apreciadas por la información que proporcionaban sobre la vida de hombres ilustres de la Antigüedad griega y latina. ${ }^{18}$ Un interés que se acrecentaba por el hecho de que Plutarco proporcionaba modelos ejemplares de conducta en un momento en el que la historia, de acuerdo con los principios ciceronianos, volvía a ser considerada como magistra vitae. El propio Heredia, consciente de la importancia de estas Vidas, encargó una traducción pionera al aragonés que fue una auténtica novedad en su momento y que llamó poderosamente la atención de humanistas italianos de la talla de Coluccio Salutati. ${ }^{19}$ Pero se da la circunstancia de que la obra biográfica de Plutarco era rica en acciones y parca en palabras. Por ello, junto al texto enciclopédico de las Vidas, el hombre bajomedieval y pre-renacentista también prestó una especial atención a una serie de obras que ofrecían una visión de lo hecho pero también de lo dicho por los héroes del pasado grecolatino que protagonizaban las biografías plutarqueas. Una predilección que explica la amplia difusión de misceláneas latinas como los Hechos y dichos memorables de Valerio Máximo, que ya era una de las lecturas preferidas de Petrarca, y que, no por casualidad, contó con traducciones tempranas a diversas lenguas vernáculas. ${ }^{20} \mathrm{El}$ interés de la obra residía en que la materia histórica había sido dividida por secciones que permitían desarrollar un cuadro de virtudes cívicas, retratos ilustres y dichos famosos que estaban, además, en clara sintonía con una concepción retórico-moralista de la instrucción. Un interés que también contribuyó a una circulación temprana de diversas colecciones de Apotegmas del propio Plutarco, entre las que se destacan sus Máximas de reyes $y$ generales, claramente concebidas como un complemento necesario al texto de las

\footnotetext{
${ }^{17}$ Ejemplos de selecciones de discursos en manuscritos de finales del siglo XIV y de comienzos del siglo XV como el Parisinus gr. 2991 A (circa 1420). Se trata de un manuscrito misceláneo en el que, entre otros textos, contiene discursos seleccionados a partir del Bellum Judaicum (los discursos de Agripa, Josefa y Tito). También contiene selecciones de las Antiquitates Judaicae (Testimonium Flavianum). O el Vindobonensis hist. gr. 113 (siglo XV), que, entre otros textos, en los ff. 151v-152r contiene el discurso de Tito en B. J. 3.472-484. O el Upsaliensis gr. 8. (siglo XV), que en los ff. 297v$299^{v}$ contiene los discursos de Tito (B. J. 3,472-484) y de Josefo (B. J. 3, 362-382).

${ }^{18}$ Sobre las traducciones de las Vidas de Plutarco, cf. Bergua Cavero (1995).

${ }^{19}$ Cf. Álvarez Rodríguez (1983).

${ }^{20}$ Cf. Avenozza (1998).
} 
Vidas y por las que se interesan humanistas de la primera época, como Francesco Filelfo, que concluye una traducción latina de estas máximas hacia 1437.

Este tipo de selecciones permitía acceder a la información proporcionada por el corpus de obras historiográficas antiguas, entendidas como una fuente inagotable de información sobre las palabras y los comportamientos de los hombres del pasado. Un objetivo que se lograba gracias a la elaboración de una estructura a partir de nacionalidades (griegos, romanos, atenienses, espartanos...) o, en todo caso, siguiendo un orden alfabético de personajes. Para comprender su finalidad es de gran interés la dedicatoria de las Máximas dirigida por Plutarco al emperador Trajano, ya que aporta una información preciosa sobre la función de esta antología de dichos tomados de la historia y permite una comparación implícita con lo que Plutarco pretendía con las Vidas. Algo especialmente interesante desde el momento en que en ambos casos se emplea la metáfora del espejo. Así, por una parte, el lector ideal de las Vidas, tal y como expone en el prólogo (Aem. 1.1-4) de las biografías de Emilio y Timoleón, usaría el "espejo" de la historia con un objetivo claramente moralizante, para modelar su propio carácter de acuerdo con las virtudes y defectos expuestos. Sin embargo, frente a la finalidad de las Vidas, el lector de estas máximas recibiría la utilidad de unas "notas" (apomnemoneumátwn) como el medio que permite comprender "el recto entendimiento de los caracteres y gustos de dirigentes, reflejados más en sus palabras que en sus hechos" (172C). Ya que, como dice más adelante, "al estar sus manifestaciones (apopháseis) y proclamas (anaphonéseis) junto a sus hechos, experiencias y fortunas, ofrecen la oportunidad de poder mirar diáfanamente como en un espejo (en katóptrois) la intención de cada uno". Estamos, por lo tanto, ante la misma metáfora del espejo pero ante finalidades bien distintas: no se trata de una vida como espejo moral en el que se mira el lector, sino del conjunto de dichos de un personaje entendido como un espejo que permite reflejar y comprender el verdadero carácter y comportamiento del personaje histórico. Estamos ante una finalidad claramente informativa: gracias a esta antología de palabras (lógous), "coleccionadas por separado (suneilegménous) como ejemplo y semilla de sus vidas" (172E), el lector ahorraba tiempo y podía comprobar de qué modo tanto el comportamiento como la oratoria son un espejo del pensamiento. ${ }^{21}$ Una finalidad que en el período estu-

\footnotetext{
${ }^{21}$ En este sentido cf. Plu. De gloria Atheniensium 345.E.6 - 345.F.3, donde el filósofo de Queroneo señala que las acciones narradas pueden hacerse más visibles para los lectores gracias al empleo de discursos, ya que, de este modo, son contempladas como a través de un espejo.
} 
diado hizo que esta obra fuera especialmente atractiva para aquellos hombres que estaban deseosos de conocer las palabras pronunciadas por esos grandes hombres de Grecia y de Roma.

Por lo tanto, a la vista de esta serie de obras clásicas que circularon durante este momento histórico, es evidente que los discursos de la Antigüedad eran vistos por los hombres cultos como el medio perfecto para adentrarse en el conocimiento del pensamiento y la oratoria de personajes históricos tan apreciados en este momento como Alejandro, César o Pompeyo. Poseer sus discursos era una manera de acercarse a las dotes de persuasión que caracterizaron a estos grandes personajes del pasado de Grecia y Roma. No es extraño, en consecuencia, que junto a estos textos enciclopédicos que eran testimonio de una manera de leer los textos históricos desde la época imperial, también circulasen códices de tipo misceláneo en los que se recogiesen discursos y cartas extraídos de las obras de los historiadores antiguos. Este tipo de excerpta facilitaba la labor de lectura de los textos históricos al ofrecer ya seleccionado lo más granado de los escritores antiguos. Un proceso selectivo que fue un aspecto fundamental en la transmisión de la obra de Salustio durante toda la Edad Media. De hecho, en algún momento de la época imperial, entre las épocas de los Flavios y de los Antoninos, los rétores ya habían elaborado y puesto en circulación una colección de discursos y de cartas extraídos de las diferentes obras del historiador romano: Bellum Catilinae, Bellum Iugurthinum e Historiae. Incluso se da el caso de que las Historiae sólo son conocidas hoy en día por medio de fragmentos, entre los que los más importantes son precisamente los discursos que fueron seleccionados para este florilegio. ${ }^{22}$ Esta antigua selección oratoria y epistolográfica vivió una amplia difusión a lo largo de toda la Edad Media hasta el Renacimiento. Por una parte, en Francia circularon copias elaboradas en Corbie ${ }^{23}$ y Auxerre ya en los siglos IX y X, donde a los discursos y cartas extraídos de las obras de Salustio se añadieron otros tipos de textos históricos. ${ }^{24} \mathrm{De}$ especial importancia es el ms. Vaticanus latinus 3864, que contiene el Comentario

\footnotetext{
${ }^{22}$ En concreto, se trata de los frg. 1.55 (Oratio Lepidi), 1.77 (Oratio Philippi), 2.47 (Oratio Cottae), 2.98 (Epistula Pompei), 3.48 (Oratio Macri), 4.69 (Epistula Mithridatis).

${ }^{23}$ Cf. Winterbotton (1983: 343): "It first appears about 790 in the palace library of Charlemagne... A copy of the book once at the palace, now lost, survives in Vatican lat. 3864, written in the third quarter of the ninth century at Corbie".

${ }^{24}$ Junto con las Invectivae, transmitidas por otros manuscritos, conforman lo que se ha denominado como Appendix Sallustiana.
} 
de Julio César sobre las Guerra de las Galias (ff. $1^{\mathrm{r}}-74^{\mathrm{r}}$ ), las Cartas de Plinio el joven (ff. $\left.78^{\mathrm{r}}-108^{\mathrm{r}}\right)$, los discursos y cartas de las obras de Salustio $\left(109^{\mathrm{r}}-127^{\mathrm{r}}\right)$ y las Epistulae ad Caesarem senem $\left(127^{\mathrm{r}}-133^{\mathrm{v}}\right)$, atribuidas al mismo autor. Por otra parte, en Italia estas selecciones fueron conocidas y utilizadas, siglos más tarde, por autores como Guglielmo da Pastrengo o Pier Candido Decembrio, ${ }^{25}$ exponentes del nuevo interés humanístico por este tipo de composiciones de cuño historiográfico. ${ }^{26}$ Esta visión de la utilidad retórica de los discursos de Salustio, entendidos como unidades oratorias independientes del lugar que ocupaban en la obra original y del sentido que el historiador quiso darle en su momento, determinó el modo en que fueron empleados como modelos retóricos, tal y como se observa en la inmensa influencia ejercida en autores esenciales de la historiografía medieval a la hora de representar las palabras de los personajes. Como señala Smalley, "that Catiline was a 'baddy', not a 'goody' makes no difference: Oratio Catilinae ad milites suos pulcherrima runs a gloss on Cat. 20. Any chronicler needing a literary model for a speech by a general encouraging his soldiers to face heavy odds had his Catilinarium to hand". ${ }^{27}$ Por este motivo, no era nada extraño que las palabras pronunciadas por Catilina, ampliamente difundidas por medio de antologías, fueran el modelo sobre el que se elaboraron los discursos puestos en boca de los más destacados reyes y generales. ${ }^{28}$

Lo ocurrido en el caso de los discursos de Salustio es una prueba del creciente interés retórico por las selecciones de discursos de origen historiográfico. Ese proceso se acrecentará a lo largo del siglo XV, extendiéndose a la obra de otros historiadores latinos como Tito Livio. Y no sólo en centros culturales de Italia, sino también en la Península Ibérica, según se puede deducir de noticias sobre obras, hoy no conservadas, como las Arengas e Propusiçiones de Enrique de Villena, las Arengues e repeticions, que había en la biblioteca de Bernat Granollach en 1468, o "las citadas en el inventario de los libros de los Condes de Benavente de Medina

\footnotetext{
${ }^{25}$ Cf. Winterbotton (1983: 348): "Pier Candido Decemblio copied the speeches of Lepidus and Philippus into his scrap-book (Milan, Ambros. R. 88 sup., ff. 98 ${ }^{\mathrm{r}}-99^{\mathrm{v}}$ )".

${ }^{26}$ El Vat. Lat. 3864 acabó en el Vaticano en 1475 y el texto de los discursos fue empleado en las primeras ediciones impresas de la obra de Salustio.

${ }^{27}$ Cf. Smalley (1971: 169-170).

${ }^{28}$ Cf. Smalley (1971: 170): "Richer puts Catiline's words into the mouth of the emperor Otto II. Bruno in his de Bello Saxonico does the same for Duke Otto adressing the Saxon rebels... Rahewin makes Frederick Barbarossa quot Catiline in a speech...”.
} 
de Pomar: Arengas e propusiçiones e abtos de los de Titu Libio". ${ }^{29}$ Una tendencia que se mantuvo a finales del siglo XV, tal y como puede comprobarse en el ms. 9-5173 de la Real Academia de la Historia en la que se conservan los Razonamientos de diferentes personajes ilustres con motivo de la guerra de Castilla y Portugal en tiempo de los reyes Don Fernando y Doña Isabel. Se trata de una antología de discursos de la Historia de Fernando del Pulgar. ${ }^{30}$

Por otra parte, ese interés por las antologías de discursos no sólo se producía en el ámbito historiográfico, sino que, con una marcada intención retórica y de manera complementaria, también se observa en otro tipo de obras que puede ayudar a entender la naturaleza de estas antologías. Nos referimos a la existencia de selecciones de discursos de tema troyano, como la que ofrece la Crónica Troyana elaborada en el escritorio de Heredia o las Orationes Homeri de Leonardo Bruni. ${ }^{31}$ En el primer caso, la selección de discursos de la Crónica Troyana de Guido delle Colonne, ${ }^{32}$ estamos ante una selección de 147 discursos, que están enmarcados en una especie de resumen narrativo de la obra original. En el último folio del manuscrito, contamos con una expresa indicación de los objetivos que se buscaban con esta selección de la Crónica Troyana (f. 194):

... porque del nuestro proposito non es tractar aquí a pleno la dicha historia, por tanto nós mandamos sacar los fundamentos et puntos de la sustancia de ella a fin que non tan solament el sentimiento de las oraciones, proposicionnes et arengas en ella contenidas millor se ofrescan entendibles a los que las leyeren, hoc encara, que cualquier pueda ayer compendioso sumario de la dicha historia por do millor pueda séller recomendada a la memoria.

Queda claro, así, que estamos ante un compendio realizado conscientemente a partir de una obra bien conocida, de donde se han "sacado" los fundamentos y puntos de la sustancia, pero, sobre todo, donde se ha prestado una especial atención

\footnotetext{
${ }^{29}$ Cf. Gómez Moreno (1996: 62).

${ }^{30}$ Cf. Carriazo (1954).

${ }^{31}$ Cf. Thierman (1993).

${ }^{32}$ La versión de Heredia ha sido estudiada y editada por Sanz Julián (2012).
} 
al "sentimiento de las oraciones, proposiciones et arengas en ella contenidas" Por otra parte, las Orationes Homeri son una traducción latina de los discursos pronunciados en el famoso episodio de la Embajada, que gozó de un gran éxito a principios del siglo XV y que, de manera casi inmediata, fue vertida al castellano por un autor anónimo. Incluso circularon copias que fueron completadas con otras composiciones de tipo oratorio, como nos muestra el ms. Holkham 339, de mediados del siglo XV, en donde, junto a las orationes homéricas de Bruni, se copiaron también varios discursos de Salustio. Puede comprobarse, por lo tanto, que los autores pre-renacentistas otorgaban a los "dichos" de personajes, ya sean históricos o épicos, un papel decisivo para conocer el glorioso pasado grecolatino, lo que permite explicar la circulación independiente de selecciones de discursos en los que alocuciones de la épica y de la historigrafía se mezclaban sin problemas. Lo verdaderamente importante era su finalidad como modelos retóricos. La pregunta que, en todo caso, hay que hacerse es la siguiente: ¿cómo se empleaban estas antologías para aprender a pronunciar discursos?

\section{Las artes arengandi o concionandi y las antologías de discursos}

Para comprender la función desempeñada por estas antologías de discursos extraídos de la historiografía o de la épica en el ámbito de la instrucción retórica de la Europa bajomedieval, consideramos que hemos de tener en cuenta el importante e influyente fenómeno que supuso, entre los siglos XII y XIII, el desarrollo en el centro y norte de Italia de un nuevo tipo de obras que van a ser ampliamente utilizadas en otros lugares de Europa: las artes arengandi o concionandi. ${ }^{34}$ Estas artes eran, al principio, la respuesta a una nueva necesidad ciudadana: la obligación de pronunciar discursos en un contexto político dominado por la creación de dos instituciones políticas: el podestà y el consejo. El primero, que solía ser un magistrado elegido entre ciudadanos sin tacha de otras comunidades, desempeñaba labores de mando y administración durante el período de un año. El segundo era una asamblea constituida por un número variable (entre cuarenta y trescientos) de ciudadanos de pleno derecho. En el desempeño de las funciones

\footnotetext{
${ }^{33}$ Cf. Gómez Moreno (1996: 62).

${ }^{34}$ Cf., al respecto, sobre todo los trabajos de Artifoni (1968), (1993), (1995) y (2001); Koch (1992); Moos (1993) y (2005) y Cammarosano (2000).
} 
encomendadas al podestà había numerosas ocasiones en las que era necesario pronunciar discursos. ${ }^{35}$

Este nuevo contexto político es uno de los factores que más contribuyeron al desarrollo de una nueva visión de cómo debía de aprenderse a pronunciar discursos y a la utilización de un modelo de oratoria basado en antologías de discursos ficticios, elaborados expresamente para atender las nuevas necesidades ciudadanas. Como es bien sabido, hasta entonces la retórica medieval había prestado atención sobre todo a la escritura de las cartas, en la que se primaba sobre todo el ámbito de la elocutio. Es decir, las artes dictandi, que comenzaron a proliferar en Europa desde el siglo XI, se centraban sobre todo en cuidar el estilo de unos textos concebidos para ser leídos. Sin embargo, desde finales del siglo XII surge la necesidad de formar a los oradores que desempeñaban diversas funciones ciudadanas en el marco del régimen de la podestà. Se produce así el florecimiento de una literatura didáctica que, en palabras de Moos, "tente de définir les règles de la communication publique et privée, en mettant l'accent aussi bien sur les aspects politiques et moraux que sur la forme de l'énnoncé et de la mise en scène". ${ }^{36}$ Esta función y este contexto explican los diversos apelativos que han recibido este tipo de manuales, entre los que se destacan los de "retórica civil" y "PodestàLiteratur". ${ }^{37}$ Lo relevante desde nuestro punto de vista es que su objetivo era proporcionar modelos concretos de elocuencia a las diversas figuras institucionales (jueces, capitanes de la guardia, etc.) que surgieron en torno a la institución de la podestà, que era un sistema político en el que se elegía a un gobernador o juez cada año y en el que se daban unas ocasiones en las que era necesario que el señor comunal o alguno de sus adjuntos pronunciasen discursos persuasivos ante el consejo, el tribunal de justicia, el pueblo o el ejército de una ciudad. Estos nuevos marcos de ejecución oratoria conllevaron la necesidad de una instrucción que preparase a los líderes ciudadanos para la pronunciación de nuevos tipos de discursos que reciben los nombres de arenga, contio o parlamentum. Era evidente que ya no bastaban las normas estilísticas de las tradicionales artes dictandi, por lo que los nuevos dictatores, que suelen ser a la vez profesores de las artes del trivium y de jurisprudencia, enseñan así el arte de ben parlare por medio de antologías de

\footnotetext{
${ }^{35}$ Cf. Cammarosano (2000: 431).

${ }^{36}$ Cf. Moos (2005: 390).

${ }^{37}$ Cf. Hetter (1910).
} 
ejemplos oratorios. ${ }^{38} \mathrm{Si}$ a este contexto político de la podestà le sumamos el hecho de que justo en aquellos años de comienzos del siglo XIII también comenzó en Italia el auge de las denominadas artes praedicandi, los manuales que formaban a un nuevo tipo de predicador más cercano a las preocupaciones de la nueva ciudadanía civil, se comprende el enorme impulso que recibió un modo de enseñar una oratoria más acorde con los nuevos tiempos. ${ }^{39}$

El nuevo método primaba el empleo de colecciones de discursos ficticios concebidos como modelos retóricos. Y no se trataba de algo totalmente nuevo. Además de la existencia de las antologías de discursos extraídos de obras épicas o historiográficas, estos nuevos procedimientos retóricos han de ponerse en relación con el hecho de que ya desde principios del siglo XIII los dictatores o maestros de retórica asociados a las escuelas de leyes habían comenzado a publicar una serie de Artes Dictaminis, en las que las explicaciones teóricas sobre cuestiones retóricas eran acompañadas por discursos y cartas elaborados para la ocasión y que servían de modelo práctico. ${ }^{40}$ Pronto, ante las nuevas necesidades ciudadanas, el interés de estos maestros se decantará hacia la composición de estos discursos civiles que reciben la denominación genérica de Arenga. En este sentido, había un amplio conjunto de obras de diversa naturaleza e intención, con ejemplos tan tempranos como el Oculus Pastoralis (1220), o los Parlamenti ed Epistole de Guido Faba (1242), obras en las que todavía se observa la mezcla de los objetivos propios tanto de la ars dictandi como de la ars arengandi, por lo que la instrucción intenta ofrecer ejemplos de ambos tipos de composiciones escritas y orales. ${ }^{41}$ Pero, sobre todo, en este caso nos interesa destacar el tipo de manual que comenzó a extenderse en Italia a partir de la segunda mitad del siglo XIII centrado de manera exclusiva en la preparación de la arenga. Es decir, en proporcionar modelos de elocuencia orales: es el triunfo de los discursos sobre las cartas. Se trata de

\footnotetext{
${ }^{38}$ Cf. Giansante (1999) y Zabbia (1999).

${ }^{39}$ Cf. Briscoe (1992).

${ }^{40}$ Cf. Witt (1982).

${ }^{41}$ Sobre la oratoria "republicana" desde finales del siglo XIII hasta finales del XV, cf. Bock (1993: 123 ss.). En concreto, cf. p. 126: "The Oculus pastoralis wich opens with a set model speeches designed for incoming podestà particularly advises them to promise that their rule will conduce "to increase and glory and honour (ad incrementum et gloriam et honorem)".
} 
las obras de Pierre de la Vigne (Arenge), ${ }^{42}$ Matteo dei Libri (Arringue), ${ }^{43}$ Filippo Ceffi (Dicerie) $)^{44}$ y Jean de Vignano (Flore de parlare).$^{45}$ En esas Artes arengandi los maestros propocionaban una mínima parte teórica con unos escuetos consejos de tipo formal y estilístico en latín, pero sobre todo ofrecían modelos de discursos ejemplares para temas tan variados como embajadas, ceremonias fúnebres o arengas militares en lengua vulgar. Distinguen por lo tanto dos niveles lingüísticos acordes con dos funciones diferentes: la breve exposición de preceptos retóricos expresada en latín y los modelos oratorios que, para lograr la mayor efectividad posible, utilizan la misma lengua vulgar en la que los oradores han de expresarse. Veamos en qué consisten una de estas antologías de discursos tomando como modelo las Arenge de Matteo de Libri. Los discursos que incluye son modelos de alocuciones de embajadores o de líderes ciudadanos (podestà). El discurso no suele aparecer completo, sino que suele consistir en un exordium y en una conclusio de contenido general, que pueden ser fácilmente adaptados por los oradores a las ocasiones concretas en las que tengan que ser empleadas. Eso significa que los discursos modelos no constituyen más que puntos de partida para la elaboración de intervenciones y que, por lo tanto, los oradores pueden abreviar, ampliar o cambiar, de acuerdo con las necesidades de cada caso, lo que estimen oportuno. Estos discursos suelen estar llenos de sentencias generales de contenido político que pueden también ser adaptadas a situaciones concretas ante las que se enfrente el orador. Así ocurre en aquellos casos en los que estas sentencias ayudan a ganar tiempo para la preparación mental de una réplica. Pero la existencia de este tipo de adagios no sólo es técnica. De hecho, es destacable que el grueso del contenido, aparte de ejemplos de salutationes o invocaciones, esté constituido por sentencias de contenido moralizante o político, en las que se habla sobre los terribles efectos de dejarse llevar por pasiones como la cólera o las nefastas consecuencias de la discordia para las ciudades.

De hecho, es interesante destacar que estas antologías de discursos no sólo tenían en cuenta la estricta utilidad retórica. En efecto, la crítica ha señalado que uno de los objetivos principales que se observan en la elaboración y difusión de estos manuales tiene que ver con la intención de propiciar un ambiente de concordia

\footnotetext{
${ }^{42}$ Cf. el texto en el anexo de Vincenti (1974: 183-227) y el estudio de Kantorowich (1909).

${ }^{43}$ Cf. el texto en Vincenti (1974) y los estudios de Kristeller (1951) y Vincenti (1969).

${ }^{44} \mathrm{Cf}$. el texto en Giannardi (1942).

${ }^{45}$ Cf. el texto en Vincenti (1974: 228-327) y el estudio de Frati (1913).
} 
ciudadana y poner fin a disputas civiles muy comunes en el nuevo contexto político. ${ }^{46}$ Precisamente este tema de la concordia es el que nos permite establecer una directa conexión entre este tipo de manuales prácticos y el influyente modelo de discurso historiográfico que proporcionaba la obra de Salustio que, como hemos estudiado, se transmitió en la Edad Media sobre todo por medio de antologías. ${ }^{47}$ De hecho, entre las autoridades antiguas citadas en estas Artes arengandi, los discursos del historiador latino son los más nombrados, hasta el punto de que uno de sus discursos, el pronunciado a favor de la concordia en Bellum Iugurthinum 10, es puesto invariablemente como modelo. Se trata del discurso que pronuncia Micipsa al morir, en el que exhorta a sus tres herederos en el trono (sus hijos Adelbal y Hiempsal y su sobrino Yugurta) a mantenerse unidos y, sobre todo, a mantener la concordia: concordia parvae res crescunt, discordia maxumae dilabuntur ("pues con la concordia se acrecientan las pequeñas cosas y con la discordia las más grandes se destruyen") (10.6). Un discurso que en la propia obra de Salustio resulta casi irónico, ya que acto seguido el historiador romano describe cómo Yugurta hace asesinar a Hiempsal y derrota y expulsa a Aderbal que ha de acudir a Roma en busca de auxilio. Pero una alocución que, en el marco de de una antología, aportaba un discurso modélico lleno de sentencias notables sobre la necesidad de mantener la paz interna entre aquellos que pertenecen a una misma comunidad. Un modelo fácilmente aplicable en el turbulento contexto italiano.

Finalmente, y con la vista puesta en Heredia, también hemos de destacar que el interés por este tipo de composiciones oratorias que tanta importancia tuvieron en la Italia de los siglos XIV y XV, tuvo un amplio eco en el Reino de Aragón. De hecho, ya un autor como Ramón Llul consideró la arenga como la forma básica de discurso en su Rhetorica nova de 1301, aportando modelos ejemplares, mientras que a finales del XIV un traductor anónimo puso en circulación una versión catalana del Tresor de Brunetto Latini. ${ }^{48}$ Pero, sobre todo, fueron los reyes aragoneses que vivieron a lo largo del siglo XIV los que estaban más familiarizados tanto con las artes arengandi como, sobre todo, con los modelos proporcionados por las artes praedicandi, como ha demostrado el detallado estudio de Cawsey. ${ }^{49}$ Una

\footnotetext{
${ }^{46}$ La conexión entre retórica y política, la mezcla de normativa retórica y de consejos sobre la administración de la ciudad, ha sido analizada por Artifoni (1968), (1993) y (1995).

${ }^{47}$ Cf. Smalley (1971).

${ }^{48}$ Cf. Johnston (1992: 106).

${ }^{49}$ Cf. Cawsey (2002: 29 ss.).
} 
influencia que todavía será perceptible en el siglo XV, en obras novelescas como el Tirant lo Blanc de Joanot Martorell, compuesta en un ámbito cultural no demasiado alejado del de Heredia, en la que se destaca el deseo de legitimar la historia ficticia de las gestas de Tirante por medio de un gran número de discursos e intervenciones en estilo directo, que muestran una clara influencia del modelo proporcionado tanto por esas artes arengandi como por los discursos de los historiadores clásicos que habían recibido el honor de circular por medio de antologías. ${ }^{50}$

\section{Conclusiones}

En el presente artículo hemos estudiado el contexto cultural y retórico de las antologías de discursos que circularon en la baja Edad Media con el objetivo de comprender la importancia y la que, desde nuestro punto de vista, es la auténtica naturaleza retórica del Tucídides de Heredia. A la vista del cuadro resultante, se pone de manifiesto que la presencia de la antología de discursos historiográficos del autor ateniense en el scriptorium del Gran Maestre en Avignon estaba en plena consonancia con las ideas contemporáneas sobre los modelos de elocuencia extraídos de la historiografía antigua y con los métodos que, a partir de las artes arengandi, eran considerados más útiles para aprenderla. Además, hay que destacar que, con el Tucídides, Heredia lograba un doble objetivo. Por una parte, contar con una antología oratoria que ofrecía modelos de discursos a partir de las palabras de los héroes de la Atenas clásica tan respetados en este momento como paradigmas de oratoria. Por otra, acceder a textos históricos de la Antigüedad, que, al estar escritos en griego o no contar con traducciones latinas, eran cada vez más apreciados por los hombres cultos del momento. Una finalidad que obsesionaba a los pre-humanistas de finales del XIV y de principios del XV, como bien sabemos por la correspondencia conservada del Gran Maestre. A la vista del contexto his-

tórico, cultural y retórico que hemos analizado, está claro que con su Tucídides el Gran Maestre consiguió alcanzar ambos objetivos.

${ }^{50}$ Cf. Pujol (1992: 59-62). 


\section{Bibliografía}

Álvarez Rodríguez, A. (1983), Las Vidas de hombres ilustres (No. 70-72 de la Biblioteca Nacional de París). Estudio y edición, tesis doctoral, Madrid.

(1986), “J. Fernández de Heredia y las traducciones del griego medieval al aragonés”, Erytheia 7: 113-131.

(2007), Tucídides, Discursos de la Guerra del Peloponeso, Zaragoza: Fundación Fernando el Católico.

Artifoni, E. (1968), "I podestà professionali e la fondazione retorica della politica comunale, Quaderni storici 63: 687-719.

(1993), "Sull'eloquenza politica nel Duecento italiano", Quaderni Medievali 35: 57-78.

(1995), "Gli uomini dell'assemblea. L'oratoria civile, i concionatori e i predicatori nella società comunale", en La predicazione dei frati dalla metà del '200 alla fine del '300, Spoleto, pp. 143-188.

(2001), "Orfeo concionatore. Un passo di Tommaso d'Aquino e l'eloquenza politica nelle città italiane nel secolo XIII", en L. Mauro (ed.), La musica nel pensiero medievale, Ravenna pp. 137-149.

Avenoza, G. (1998), "La recepción de Valerio Máximo en las Coronas de Castilla y de Aragón en el medievo", Euphrosyne 26: 241-252.

Banker, J. R. (1974), "The Ars dictaminis and Rhetorical Textbooks at the Bolognese University in the Fourteenth Century", Mediaevalia et Humanistica 5: 153-168.

Benson, R. B. L. (1977), "Protohumanism and Narrative Technique in Early Thirteenth Century Italian «Ars dictaminis»", en M. Cottino-Jones et al. (eds.), Boccaccio: Secoli di vita, Ravenna, pp. 31-50.

Bergua Cavero, J. (1995), Estudios sobre la tradición de siglos XIII-XVII, Zaragoza: Universidad de Zaragoza. 
Bock, G., Skinner, Q. y Viroli, M. (eds.). (1993), Macchiavelli and Republicanism, Cambridge: University Press.

Bonner, S. F. (1966), "Lucan and the Declamation Schools" American Journal of Philology 87: 257-89.

Briscoe, M. G. y Jaye, B. H. (1992), Artes praedicandi / Artes orandi (Typol. des sources du Moyen Âge occidental fasc. 61), Turnhout.

Brunhölzl, F. (1986), “Curtius Rufus im Mittelalter”, Lexikon des Mittelalters, vol. 3, col. 393.

Cacho Blecua, J. M. (1997), El Gran Maestre Juan Fernández de Heredia, Zaragoza: Caja de Ahorros de la Inmaculada.

Cammarosano, P. (2000), "L'eloquence laïque dans l'Italia communales (fin du XIIe-XIVe siècle)", Bibliothèque de l'École des Chartres 158: 431-442.

Carriazo, J. de Mata (1954), "Las arengas de Pulgar", Anales Universidad Hispalense 15: 43-74.

Cawsey, S. F. (2002), Kingship and Propaganda. Royal Eloquence and the Crown of Aragon 1220-1450, Oxford: University Press.

Coroleu, A. (2001), "Josefo, historiador de tanto crédito: Translations of Josephus in Renaissance Iberia", Euphrosyne 19, 185-192.

(2004), "A Preliminary Survey of Greek and Latin Historians in Translation in the Iberian Peninsula (c.1360-1599)", Bulletin of Hispanic Studies 81, 897-912.

Dosson, S. (1887), Étude sur Quinte Curce. Sa vie et sa oeuvre, París: Hachette.

Frati, C. (1913), "Flore de parlare o Somma d'arengare attribuita a Ser Giovanni fiorentino da Vignano in un codice marciano", Giornale Storico della Letteratura italiana 61: 1-31 y 62: 228-265.

Galletti, A. (1938), "L'eloquenza (dalle origini al XVI secolo)", en Storia dei generi letterari Italiani, Milán, vol. 2, pp. 411-597. 
Gaudenzi, A. (1895), "Sulla cronologia delle opere dei dettatori bolognesi da Buoncompagno a Bene di Lucca", Bullettino dell'istituto storico italiano 14: 90-118.

Giannardi, G. (1942), “Le 'Dicerie' di Filippo Ceffi”, Studi di Filologia Italiana 6: 5-63.

Giansante, M. (1999), Retorica e politica nel Duecento: I notai bolognesi e l'ideologia comunale, Roma.

Gómez Moreno, A. (1996), (1996), “Juan Fernández de Heredia, ¿Humanista?”, en A. Egido y J. M. Enguita (eds.), Juan Fernández de Heredia y su Época, Zaragoza: Institución Fernando el Católico, pp. 57-68.

Hetter, F. (1910), Die Podestàliteratur Italiens im 12. und 13. Jhs., Leipzig-Berlin.

Iglesias-Zoido, J. C. (2005), "El Tucídides de Juan Fernández de Heredia: Problemas planteados por la selección de discursos", $A E F, 28:$ 131-147.

(2008), "El Tucídides de Heredia en el contexto retórico del siglo XIV", en J. M. Maestre, J. M. Pascual y L. Charlo (eds.), Humanismo y Pervivencia del Mundo Clásico IV: Homenaje al profesor Antonio Prieto, Alcañiz y Madrid: Instituto de Estudios Humanísticos y CSIC, vol. II, pp. 941-956.

(2011), El legado de Tucídides en la cultura occidental: Discursos e historia, Coimbra: Classica Digitalia.

(2015), "The Speeches of Thucydides and the Renaissance Anthologies", N. Morley (ed.), A Handbook of the Reception of Thucydides, Londres y N. York: Willey-Blackwell (en prensa).

Johnston, M. D. (1992), "Parliamentary Oratory in Medieval Aragon”, Rhetorica 10: $99-118$.

Kantorowicz, H. U. (1909), "Über die dem Petrus von Vinea zugeschriebenen Arenge", Mitteilungen des Instituts für Österr. Geschichtsforschung 30: 651-654. 
Koch, P. (1992), “Ars arengandi”, en G. Ueding (éd.), Historisches Wörterbuch der Rhetorik, vol. I, cols.1033-1044.

Kristeller, P. O. (1951), "Matteo de' Libri, Bolognese Notary of the Thirteenth Century, and his Artes Dictaminis", en Miscellanea G. Galbiati II, Milán, pp. 283-320.

(1983), "Rhetoric in Medieval and Renaissance Culture", en J. J. Murphy (ed.), Renaissance Eloquence, Berkeley-Los Angeles, pp. 1-19.

Lake, J. (2013), Richer de Saint-Remi, The Methods and Mentality of a TenthCentury Historian, Cambridge: University Press.

López Molina, L. (1961), Tucídides romanceado en el siglo XIV, Madrid: Anejos RAE.

Lutrell, A. (1960), “Greek Histories translated and compiled for Juan Fernández de Heredia, Master of Rhodes, 1377-1396”, Speculum 35: 401-407.

Moos, P. von (1993), “Aspekte der Dialogforschung, Die italienische ars arengandi des 13. Jahrhunderts", en H. Brunner y N. R. Wolf(eds.), Wissensliteratur im Mittelalter und in der Frühen Neuzeit, Wiesbaden, pp. 67-90.

(2005), "L' Ars arengandi italienne du XIIIe siècle: une école de la communication", en Entre histoire et litterature: communication et culture au Moyen Âge, Florencia: Sismel, pp. 389-415.

Munk Olsen, B. (1982), L'étude des auteurs classiques latins aux XIe et XIIe siècles (Documents, Études et Répertoires), vol. I, París: Institut de Recherche et d'Histoire des Textes.

Osmond, P. y Ulery, R. (2003), "Sallustius Crispus, Gaius", in Catalogus translationum et commentariorum, vol. 8, ed. Virginia Brown, Washington, pp. 183-326.

Pade, M. (2003), (2003), “Thucydides”, en V. Brown (ed.), Catalogus Translationum et Commentariorum, vol. VIII, Washington: The Catholic University of America Press, pp. 103-182. 
(2006), “Thucydides' Rennaissance Readers”, en A. Rengakos y A. Tsatmakis (eds.) (2006), Brill's Companion to Thucydides, Leiden: Brill, pp. 779-810.

Pujol, J. (1992), La memòria literària de Joanot Martorell. Models i escriptura en el Tirant lo Blanc, Barcelona: Curial.

Rubinstein, N. (1945), "Political Rhetoric in the Imperial Chancery during the Twelfth and Thirteenth Centuries", Medium Aevum 14: 21-43.

Sanz Julián, M. (ed.) (2012), Crónica troyana de Juan Fernández de Heredia, Zaragoza: Prensas Universitarias de Zaragoza.

Segre, C. (1968), "Le forme e le tradizioni didattiche", en Grundriss der Romanischen Literaturen des Mittelalters VI: La littérature didactique, allégorique et satirique, Heidelberg, vol. I, pp. 58-145 y vol. II, pp. 297-201.

Smalley, B. (1971), "Sallust in the Middle Ages", en Bolgar (ed.), Classical Influences on European Culture (A.D. 500-1500), Cambridge: University Press, pp. $165-175$.

Sorbelli, A. (1944), "I teorici del Reggimento comunale", Bullettino dell'Istituto storico italiano per il medioevo e Archivio Muratoriano 59: 31-136.

Thierman, P. (1993), Die Orationes Homeri des Leonardo Bruni Aretino, Leiden: Brill.

Ussani, V. (1932), Hegesippi qui dicitur historiae libri V, (Corpus Scriptorum Ecclesiasticorum Latinorum vol. 66), Vienna: Hölder-Pichler-Tempsky.

Vecchi, G. (1960), “Le arenge di Guido Faba e l'eloquenza d'arte civile e politica duecentesca", Quadrivium 4: 61-90.

Vincenti, E. (1969), "Matteo dei Libri e l'oratoria pubblica e privata nel '200", Archivio glottologico italiano 54: 227-237.

(ed.) (1974), Matteo dei Libri, 'Arringhe’, Milán y Nápoles.

Ward, J. (1972), Artificiosa Eloquentia in the Middle Ages, Ph. D., Toronto. 
Weiss. R. (1977), Medieval and Humanist Greek, Padua: Antenore.

Winterbotton, M. (1983), "Curtius Rufus", en Reynolds (ed.), Texts and Transmission: A Survey of the Latin Classics, Oxford: University Press, pp. 148-149.

Witt, R. (1982), "Medieval Ars Dictaminis and the Beginning of Humanism", Rennaisance Quarterly 35, 1-35.

Zabbia, M. (1999), I Notai e la cronachistica cittadina italiana nel Trecento, Roma. 